\title{
Cluster Analysis of Center-of-Pressure Measures
}

\author{
Shabana Baig, Richard M. Dansereau, Adrian D. C. Chan \\ Department of Systems and Computer Engineering, Carleton University \\ 1125 Colonel by Drive, Ottawa, ON K1S 5B6 - Canada \\ sbaig@sce.carleton.ca; rdanse@sce.carleton.ca; adcchan@sce.carleton.ca \\ Anthony Remaud, Martin Bilodeau \\ School of Rehabilitation Sciences, Faculty of Health Sciences, University of Ottawa; and \\ Bruyère Continuing Care \\ Élisabeth Bruyère Research Institute \\ 43 Bruyère Street, Ottawa, ON K1N 5C8 - Canada \\ aremaud@bruyere.org; martin.bilodeau@uottawa.ca
}

\begin{abstract}
Postural stability analysis is useful in a variety of applications, such as assessing the risk of falls for older adults or investigating neuromuscular disorders. Variations in the center-of-pressure (COP) are often used to assess postural stability. The COP is a point where the vertical reaction forces of the ground act. It represents the weighted average of all pressures over the body in contact with the ground. Static posturography, which analyses COP variations during a fixed standing posture, is a non-invasive assessment technique, used to quantify postural stability. The objective of this study is to investigate the performance of six conventional COP measures used to characterize postural stability. Six conventional measures are investigated: i) average radial displacement, ii) average velocity, iii) area of the 95\% confidence ellipse, iv) standard deviation of COP in the $x$-direction (medio-lateral), $v$ ) standard deviation of COP in the y-direction (antero-posterior), and vi) standard deviation of radial displacement. These COP measures are compared during four different stability conditions: i) feet together, eyes open, ii) feet together, eyes closed, iii) single leg, eyes open, and iv) single leg, eyes closed. Performance is quantified by cluster analysis using the silhouette coefficient, which provides a measure of how well clustered the four stability conditions are for a given stability measure. A good stability measure should have high repeatability for a given stability condition (low intra-cluster distances) and be able to discern between different stability conditions (high inter-cluster distances). Results from eight subjects suggest that out of the six COP measures examined, average velocity is the best measure to assess postural stability.
\end{abstract}

Keywords: Center-of-Pressure, Sway Path, Posturography, Postural Stability, Silhouette Coefficient
(C) Copyright 2012 Authors - This is an Open Access article published under the Creative Commons Attribution License terms (http://creativecommons.org/licenses/by/2.0). Unrestricted use, distribution, and reproduction in any medium are permitted, provided the original work is properly cited.

\section{Introduction}

Postural control refers to maintaining balance and orientation in the gravitational field, which is essential for the performance of everyday activities such as walking or reaching for objects. Postural control is a complex phenomenon where equilibrium of the body must be maintained in the gravitational field by continuous adjustments of center-of-pressure (COP) within the base support (Horak, 1987) (Dietz, 1992). The control system receives information from various afferent systems and integrates this information with the central nervous system to adjust posture and related muscle contractions (Dietz, 1992). Collins and De Luca (1993) believe that in addition to the above closed loop mechanisms; the postural control system also employs open-loop control schemes where the open loop activation signal results in small mechanical fluctuations at various joints of the body.

Postural stability depends on a multisensory system (e.g., vestibular, visual, and somatosensory), motor components of the nervous system (Massion, 1994), and the cognitive system (Askvik, 2010). When the condition of these systems change due to ageing or disease, body sway often increases, as does the activity of the muscles used to maintain postural balance (Massion, 1994). Studies have shown that postural sway for individuals after a stoke can become twice as high as their healthy aged-matched peers (Nichols, 1997). 
Measurements of postural stability are used to identify neuromuscular diseases (Schmit et al., 2006) and to assess the risk of falls in frail populations (Jbabdi, Boissy, \& Hamel, 2008). Studies have also shown that people with neuromuscular disorder (e.g., polio survivors) demonstrate a higher frequency of falls, frequently resulting in injury and subsequent fear of falling (Bickerstaffe, Beelen, and Nollet 2010). The fear of falling is an important factor in restriction of physical activities, which results in lower income, deprivation on healthy activities and depression (Curcio, Gomez, \& Reyes-Ortiz, 2009).

Thirty to sixty percent of the senior population (65 years or older) fall every year (Jbabdi et al., 2008). Consequences of falls in seniors can include an increase in mortality, decline in mobility, and other health complications that decrease quality of life and require early geriatric care (American Geriatrics Society, British Geriatrics Society, and American Academy of Orthopaedic Surgeons Panel on Falls Prevention, 2001). A fall happens when stability is adequately disturbed, and time control and muscular strength are not sufficient to recover or adapt to these perturbations. Aging affects the forces used to control posture (Mcclenaghan et al., 1996). There are many additional age-associated factors contributing to a decline in stability, such as balance or gait disorders (Lord, Menz, \& Tiedemann, 2003), and cognitive impairments, particularly a misperception of orientation (Jensen, Nyberg, Gustafson, \& Lundin-Olsson, 2003). Falls and fall associated fractures are the major cause of disability in seniors (Shimada et al., 2011). Early intervention can help to mitigate the risk of falling, and related health and social issues (Shimada et al., 2011). All this motivates researchers and clinicians to develop new ways to understand and quantify postural stability.

There are a number of existing methods, technical and nontechnical, for the assessment of postural stability. Nontechnical methods, such as the Berg balance scale (Berg, Maki, Williams, Holliday, \& Wood-Dauphinee, 1992), the functional reach test (Duncan, Weiner, Chandler, \& Studenski, 1990), and the fall risk index (Tinetti, Williams, \& Mayewski, 1986), are easy to conduct and do not need expensive equipment (Browne \& O'Hare, 2000a). However, these methods may not be sensitive enough to measure minor improvement or deterioration in an individual's postural stability (Browne \& O'Hare, 2000a). Technical methods, such as posturographic analysis, are used to provide a quantitative assessment of postural stability and are relatively easy to conduct (Baratto, Morasso, Re, \& Spada, 2002) (Browne \& O'Hare, 2000a). With posturography, an individual's COP trajectory is typically recorded by using a force platform. The posturograph (see Fig. 1(c)) is a two dimensional plot of the antero-posterior (AP) (Fig. 1(a)) and medio-lateral (ML) (Fig. 1(b)) displacement of the COP (Baratto et al., 2002). The COP trajectory reflects the dynamic nature of postural control. In static posturography, the COP displacements, occurring during static posture are analyzed. Although the unperturbed upright stance position looks static, there exists a process of rocking from the ankle; this rocking or swaying is often compared with a simple inverted pendulum (Gage, Winter, Frank, \& Adkin, 2004). This low-amplitude swaying can be indexed by the COP (Pellecchia \& Shockley, 2005).

(b)

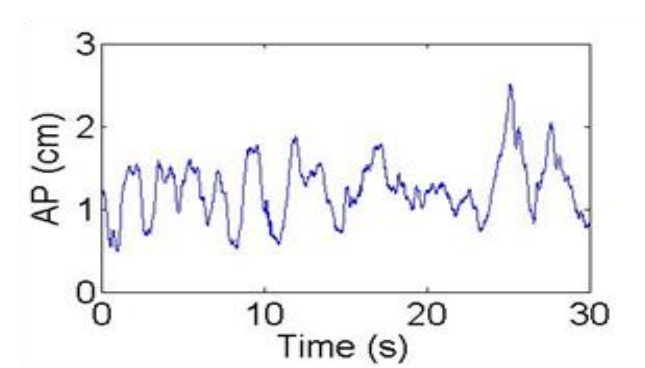

(c)
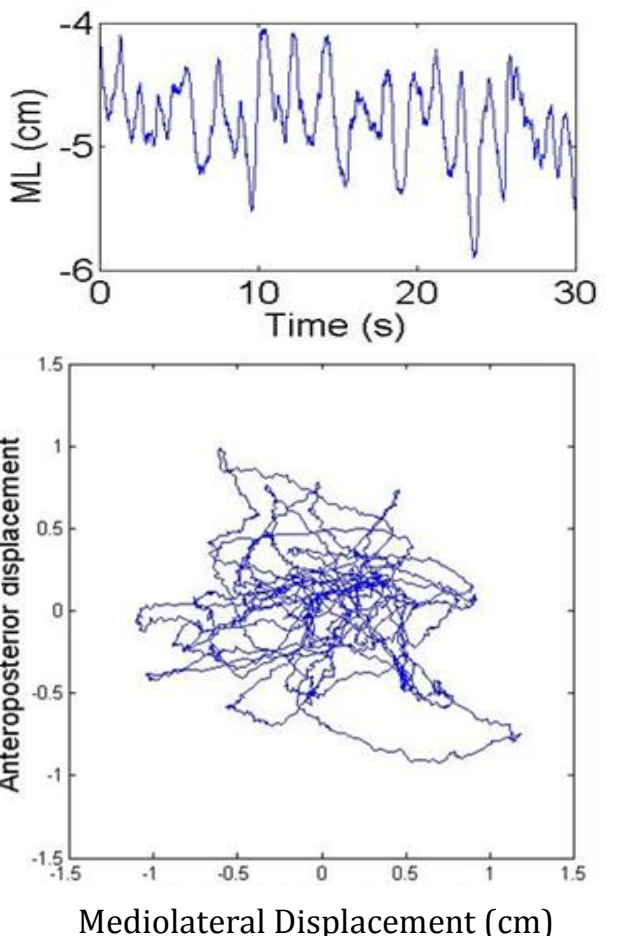

Fig. 1. Static posturograph for subject \#2, trial 1 with feet together and eyes closed (FT-EC). Plotted are the last $30 \mathrm{~s}$ of a $60 \mathrm{~s}$ recording: (a) antero-posterior (AP) displacement of the COP versus time, (b) medio-lateral (ML) displacement of the COP versus time, and (c) antero-posterior versus medio-lateral displacement, with mean $\mathrm{COP}_{x}$ and $\mathrm{COP}_{y}$ subtracted for AP and $\mathrm{ML}$, respectively to center at $(0,0)$.

The dynamic nature of COP displacements helps us to understand the inherent complexity of the postural control system and its constituent processes (Winter, 1995). The analysis of the COP motion gives comprehensive information of postural stability. Conventional COP measures used to quantify postural stability include the average radial displacement of the COP (Gosselin, Rassoulian, \& Brown, 2004), the average velocity of the COP (Gosselin et al., 2004), area traversed by the COP (Cavalheiro, Almeida, Pereira, \& 
Andrade, 2009), standard deviation of the $C O P_{x}$ (ML) , standard deviation of the $C_{0} P_{y}$ (AP) (Doyle, Hsiao-Wecksler, Ragan, \& Rosengren, 2007) (Clair \& Riach, 1996) and standard deviation of the radial COP displacement (Prieto, Myklebust, Hoffmann, Lovett, \& Myklebust, 1996). Such variables have been shown to be sensitive to various health conditions. For example, low back pain patients have been reported to exhibit greater COP excursions and a higher mean velocity as compared to healthy individuals (Ruhe, Fejer, \& Walker, 2011).

There are numerous COP measures; however it is not evident which measure is optimal (Chaudhry, Bukiet, Ji, \& Findley, 2011), even if the average velocity of the COP tends to be a more sensitive measure (Raymakers, Samson, \& Verhaar, 2005). A good COP measure should be able to differentiate between levels of stability, while simultaneously being repeatable for the same level of stability. In this study the performance of COP measures, in the sense of repeatability and differentiability for different levels of stability conditions, are assessed by employing cluster analysis. This work is an extension of the conference paper presented by the authors in Baig et al. (2012) with an increased experimental study and introduction of noise analysis of the experimental setup.

\section{Clustering and the Silhouette Coefficient}

Cluster analysis was used to quantify the performance of COP measures in the sense of repeatability and differentiability between different stability conditions. For a given subject, the repeated trials were used to form clusters of COP measures, clustered by stability condition. A good stability measure will maximize the inter-cluster distances (increased ability to differentiate between different levels of stability) and minimize the intra-cluster distances (increased repeatability for a given level of stability). To quantify the inter- and intra-cluster distances the silhouette coefficient (Rousseeuw, 1987) was used.

The silhouette coefficient calculates the silhouette width for each sample, from which the average silhouette width for each cluster and overall average silhouette width for a total data set can be computed. The silhouette coefficient for a single object $x$ is defined as (Rousseeuw, 1987)

$S(x)=\frac{b(x)-a(x)}{\max \{a(x), b(x)\}}$

where $a(x)$ is the average distance of object $x$ to all other objects in the same cluster and $b(x)$ is the average distance of object $x$ to objects in the next nearest cluster. An average silhouette width, for either a cluster or the total data set, can be computed as

$S C=\frac{1}{M} \sum_{i=1}^{M} S\left(x_{i}\right)$

where $x_{i}$ is an object being considered, $i=1, \ldots, M$.
The silhouette coefficient $S(x)$ lies between -1 and 1 ; values close to 1 indicate that clusters of COP measures are compact and well-separated for different stability conditions, whereas values that are close to -1 indicate that clusters are not well-separated and may overlap. If the value is zero then the clusters for different stability conditions will not be differentiable. Therefore, a larger silhouette coefficient suggests better performance of a COP measure.

\section{Methods \\ 3.1 Noise Analysis}

A force platform is highly susceptible to different kinds of noise; to ensure the reliability of the various measures of $\mathrm{COP}$, the baseline noise and precision of the force plate were investigated. To collect the noise data, the force platform was divided into a grid of $4 \times 4$ squares. A load of $45 \mathrm{~kg}$ was applied on the force platform at five different positions (center $c$, front $f$, back $b$, right $r$, and left $l$ ) as shown in Fig. 2(a) with photos of each setup shown respectively in Fig. 2(b). The values of COP were calculated for $120 \mathrm{~s}$ of data for each position with results tabulated in Table 1 . The noise in the force platform signal due to a static load on the force platform is a background measurement noise and noise induced due to the positioning of a load (or a person) on the force platform is a spatial noise (Browne \& O'Hare, 2000a, 2000b). Ideally, the value of COP should be same in spite of where the subject is standing or where the load is placed on the surface of platform.

(a)

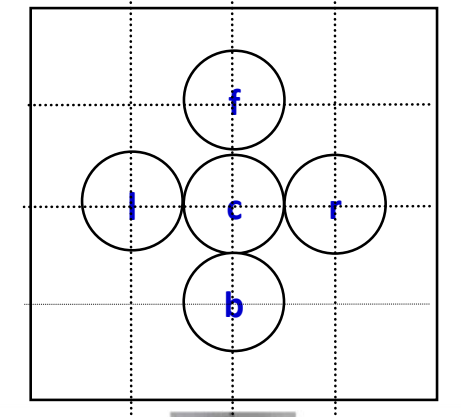

(b)

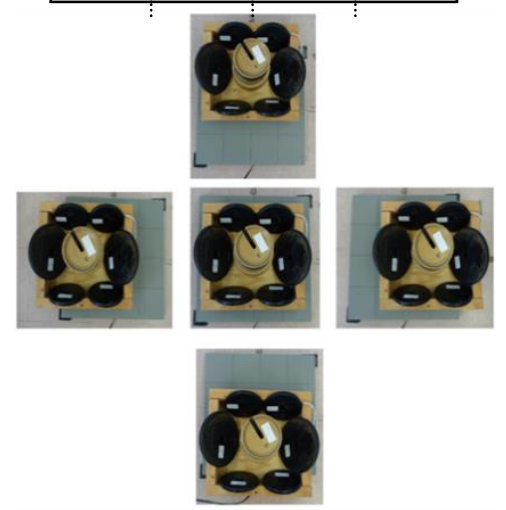

Fig. 2. (a) Diagram of the co-ordinate of interest for placing load on surface of force platform, (b) photos of loads on the force platform on five different positions; center $c$, front $f$, back $b$, right $r$, and left $l$ for the noise analysis. 


\subsection{Data Acquisition}

This research was reviewed and approved by the Research Ethics Boards (REB) of Bruyère Continuing Care and the University of Ottawa. Data were collected from eight subjects, five males $(25,31,31,33,45$ years of age $)$ and three females (21, 25, 48 years of age). All subjects had no known postural stability problems. For each subject the data were collected in one session. Each participant performed 11 trials of each of four postural stability conditions: 1 ) feet together, eyes open (FT-E0), 2) feet together, eyes closed (FT-EC), 3) single leg, eyes open (SL-EO), and 4) single leg, eyes closed (SL-EC). For these two last conditions, participants used their preferred leg, i.e. the one that they felt most comfortable using when standing on a single leg. The order of the four stability conditions was randomized to create 11 sets of the four stability conditions. The order of sets was also randomized between subjects. Sixty seconds of data were recorded for each stability condition, with $60 \mathrm{~s}$ of rest between stability conditions. A rest of $120 \mathrm{~s}$ was introduced between each set of four stability conditions to avoid muscle fatigue. An Advanced Mechanical Technology, Inc. (AMTI) force platform (AccuGait System, Watertown, MA, USA) was used to record the forces and moments in the $x, y$, and $z$ directions; these forces and moments were used to calculate the COP. Data were sampled at a sampling frequency of 100 $\mathrm{Hz}$ using a 16-bit A/D converter and were processed offline in Matlab (MathWorks Inc., Natick, MA, USA).

\subsection{Data Analysis}

Although $60 \mathrm{~s}$ of COP data were recorded, only the last $30 \mathrm{~s}$ of data for a given trial were used in the analysis. Preliminary analysis of the data suggested that subjects were still establishing their static posture during the initial $30 \mathrm{~s}$. Assuming that the data is normally distributed, the mean COP position was subtracted for each recording, such that the resulting COP was centered at zero in both the ML and AP directions. Six conventional measures of postural COP were computed for this study: 1) average radial displacement $\left(R D_{\text {avg }}\right.$ ) (Gosselin et al., 2004), 2) average velocity ( $\left.V_{\text {avg }}\right)$ (Gosselin et al., 2004), 3) area of the 95\% confidence ellipse (AREA) (Cavalheiro et al., 2009), 4) standard deviation of $\left.\operatorname{COP}_{x}\left(\sigma_{x}\right), 5\right)$ standard deviation of $\operatorname{COP}_{y}\left(\sigma_{y}\right)$ (Doyle et al., 2007) (Clair \& Riach, 1996), and 6) standard deviation of radial displacement ( $\left.s d \_R D\right)$, (Prieto et al., 1996). Additional details regarding the calculation of these COP measures are provided in the Appendix. These measures were clustered and analyzed separately for each subject and each measure by calculating the overall average silhouette coefficient width over 11 trials and all (four) stability conditions to find the best measure. We define the best measure as exhibiting a higher average silhouette coefficient, resulting in a measure that is more repeatable within the given stability cluster and discernible between the different stability clusters. In the statistical analysis, a repeated measures ANOVA and post-hoc tests using the Bonferroni correction were performed for each subject to find out whether the best selected measure was also significantly best among the investigated measures.

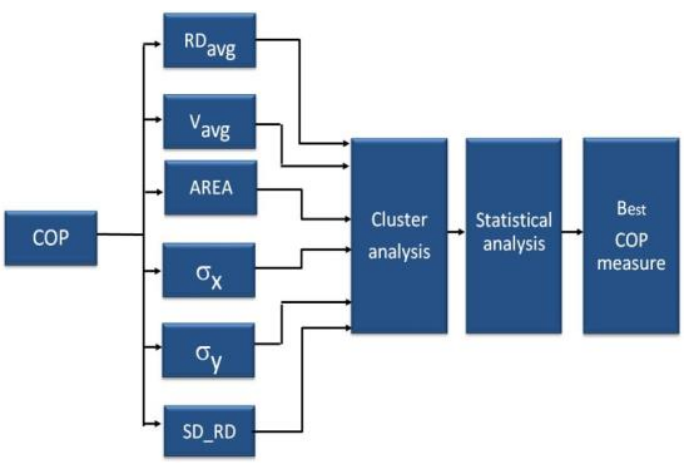

Fig. 3. Flow diagram of analysis methodology.

The significance level was set at 0.05 . Figure 3 represents the overall methodology followed to find the best measure in the sense of repeatability and discernability. To quantify the inter- and intra-cluster distances, the silhouette coefficient (Rousseeuw, 1987) was calculated. A larger silhouette coefficient will suggest better performance of a COP measure.

\section{Results \\ 4.1 Noise Analysis}

Table 1 presents the maximum COP displacement for the statically positioned test load. The maximum COP displacement recorded was $0.737 \mathrm{~mm}$ for the center position of the load. Differences in $\mathrm{COP}_{\max }$ for the other positions were less than $0.031 \mathrm{~mm}$, suggesting that spatial noise is inconsequential for the given force platform used. All other variations in the background noise are less than $0.737 \mathrm{~mm}$. Thus, the results confirm that the background and spatial noises are inconsequential according to the Association Française de Posturologie (Browne \& O'Hare, 2000b). Therefore, the experimental setup was verified to be suitable for COP performance analysis.

Table 1. The maximum COP values in noise measurements.

\begin{tabular}{|c|c|c|c|c|c|}
\hline Background & \multicolumn{5}{|c|}{ Maximum Displacement (mm) } \\
\cline { 2 - 6 } Noise & Center & Left & Right & Front & Back \\
\hline COP $_{\max }$ & 0.737 & 0.706 & 0.736 & 0.732 & 0.726 \\
\hline
\end{tabular}

\subsection{Performance of COP Measures}

Figure 4 shows a box plot of the clusters of COP measures for subject \#2; other subjects exhibited similar behaviours. As expected, the values of each measure increase from FT-EO, to FT-EC, to SL-EO, to SL-EC, suggesting a decrease in postural stability through these postures. For $V_{\text {avg }}$, most of the values in the clusters are closer to the median (i.e., they are repeatable). 


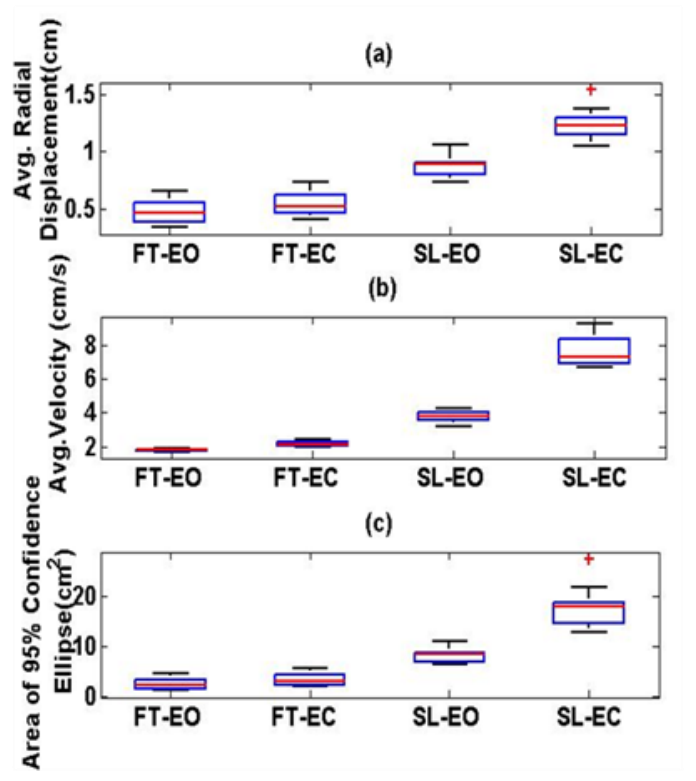

(d)

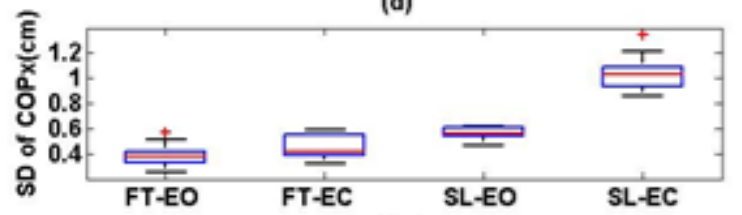

(e)
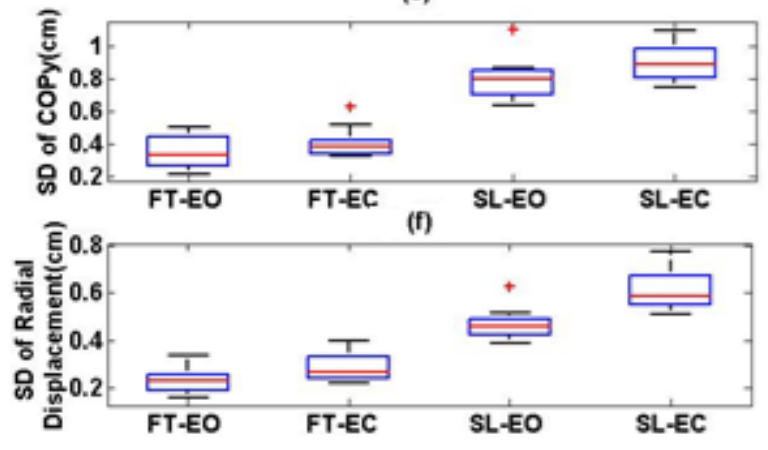

Fig. 4. Box plot of clusters of conventional COP measures for the 11 trials of subject \#2: (a) average radial displacement (RDavg), (b) average velocity ( $\left.V_{\text {avg }}\right)$, (c) area of 95\% ellipse traversed by COP $(A R E A)$, (d) standard deviations of COP $\mathrm{x}_{\mathrm{x}}\left(\sigma_{\mathrm{x}}\right),(\mathrm{e})$ standard deviations of $\mathrm{COP}_{y}\left(\sigma_{y}\right)$, and (f) standard deviations of radial displacement $\left(S D_{-} R D\right)$.

(a) Subject 1

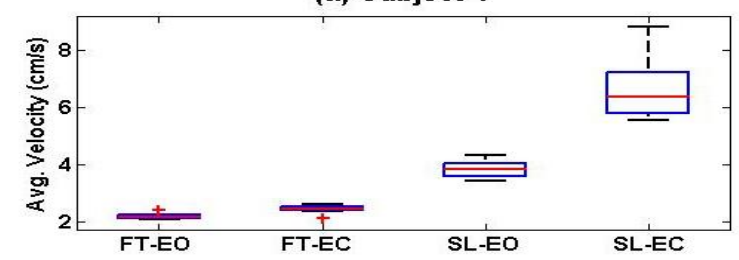

(b) Subject 2

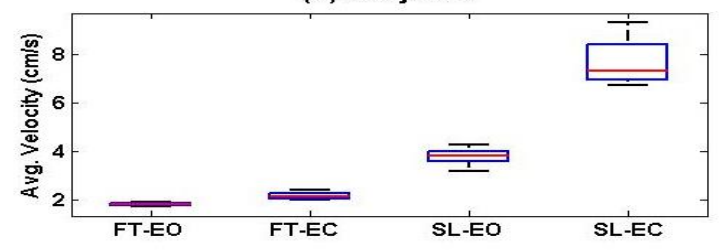

(c) Subject 3

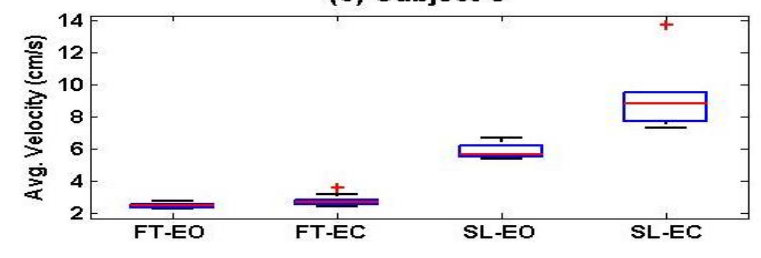

(d) Subject 4

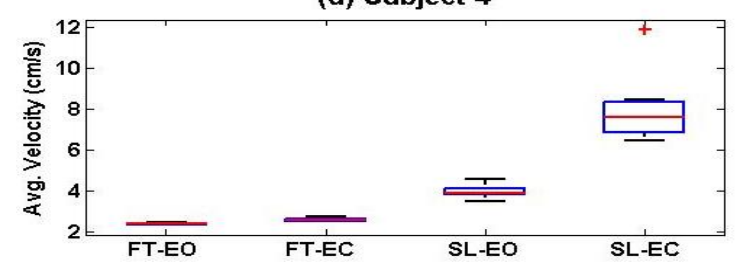

(e) Subject 5

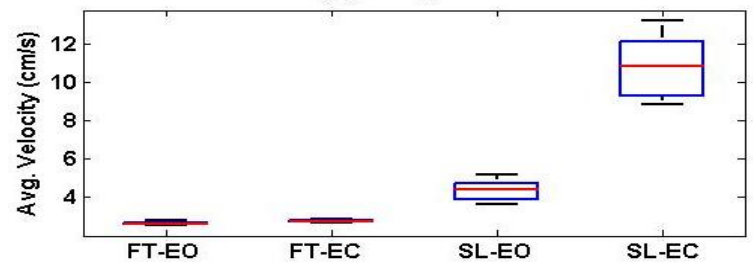

(f) Subject 6

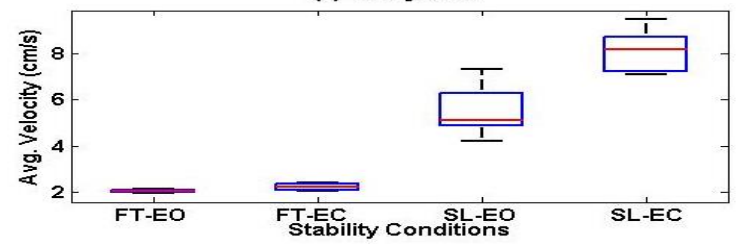

(g) Subject 7

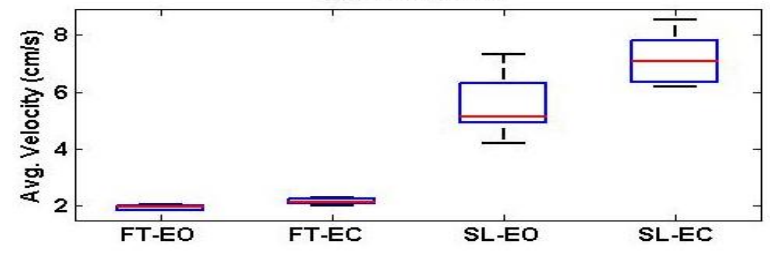

(h) Subject 8

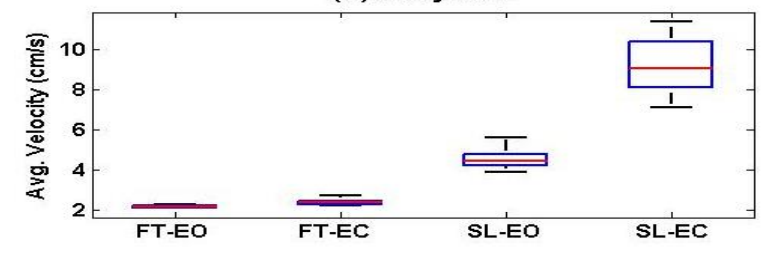

Fig. 5. Box plot of clusters of average velocity ( $\left.V_{a v g}\right)$ for subjects \#1 to \#8. 
These box plots also show that the clusters are wellseparated compared to other measures. For $V_{\text {avg }}$, clusters do not appear to overlap and are more separated than other COP measures, suggesting that the different stability conditions are more easily discerned. Figure 5 depicts the same performance of the average velocity for all eight subjects where a similar level of clustering is seen for each stability condition for all eight subjects.

To further investigate the COP measures, the silhouette coefficients were calculated for all subjects and for the six COP measures; where higher values are desirable. Figure 6 shows a bar graph of the silhouette coefficients for all COP measures, for all eight subjects. It is clear from Fig. 6 that the silhouette coefficients for $V_{\text {avg }}$, for all subjects, are much higher than the silhouette coefficients for other COP measures. Many of the COP measures have negative silhouette coefficients for some subjects, indicating that clusters overlap and are not well separated; the smallest silhouette coefficient for $V_{\text {avg }}$ is 0.28 for subject \#3 from the possible range of -1 to 1 .

Since the silhouette coefficient values for $V_{\text {avg }}$ appear significantly larger than other COP measures, a statistical analysis was performed. A repeated measure ANOVA with a single factor was used. The significant result $F(5,35)=49.69$ ( $p$ $<0.001$ ) signifies that at least two of the silhouette coefficients are significantly different. To find out if the silhouette coefficient for $V_{\text {avg }}$ is significantly larger than the silhouette coefficient for other measures, post-hoc tests using the Bonferroni correction were conducted with results presented in Table 2.

Table 2. Pairwise comparison using Bonferroni post-hoc test.

\begin{tabular}{|c|c|c|c|c|c|}
\hline \multirow{2}{*}{$\begin{array}{l}\text { COP Measures } \\
\text { Pair }\end{array}$} & \multirow{2}{*}{$\begin{array}{c}\text { Mean } \\
\text { Difference } \\
\text { of COP } \\
\text { Measure } \\
\text { Pair }\end{array}$} & \multirow{2}{*}{$\begin{array}{l}\text { Std. } \\
\text { Error }\end{array}$} & \multirow[t]{2}{*}{ Sig. ${ }^{a}$} & \multicolumn{2}{|c|}{$\begin{array}{l}\text { 95\% Confidence } \\
\text { Interval for } \\
\text { Difference }^{\mathrm{a}}\end{array}$} \\
\hline & & & & \begin{tabular}{|l|} 
Lower \\
Bound \\
\end{tabular} & $\begin{array}{l}\text { Upper } \\
\text { Bound }\end{array}$ \\
\hline$V_{a v g}-R D_{a v g}$ & $0.351^{*}$ & 0.039 & 0.001 & 0.182 & 0.520 \\
\hline$V_{a v g}-A R E A$ & $0.369^{*}$ & 0.033 & $<0.001$ & 0.223 & 0.514 \\
\hline$V_{a v g}-\sigma_{x}$ & $0.390^{*}$ & 0.044 & 0.001 & 0.198 & 0.582 \\
\hline$V_{a v g}-\sigma_{y}$ & $0.443^{*}$ & 0.052 & 0.001 & 0.218 & 0.667 \\
\hline$V_{a v g}-S D_{-} R D$ & $0.432^{*}$ & 0.032 & $<0.001$ & 0.293 & 0.571 \\
\hline
\end{tabular}

The post-hoc test revealed that silhouette coefficients for $V_{\text {avg }}$ were significantly larger than all the compared COP measures $(\alpha=0.05)$. The silhouette coefficient for $V_{\text {avg }}$ was the largest for each subject, indicating that clusters for $V_{\text {avg }}$ were well separated and compact compared to other measures.

\section{Discussion}

The silhouette coefficient for $V_{\text {avg }}$ was the largest amongst the COP measures examined. This implies that clusters of $V_{\text {avg }}$ were well separated, which specifies that the inter-cluster distances were maximum and intra-cluster distances minimum as a whole compared to the clusters of other COP measures examined.

From Fig. 6, the values of the silhouette coefficients for $V_{\text {avg }}$ compared to the silhouette coefficients for the other measures is clearly higher, the post hoc test in Table 2 show that the silhouette coefficients for $V_{\text {avg }}$ are significantly higher $(\alpha=0.05)$. It is noted from Fig. 6 that other measures may also allow for reasonable clustering for some subjects, such as $R D_{\text {avg }}$ and $A R E A$ for subjects $\# 2$, \#4, and \#5, where the silhouette coefficients approach 0.3. However, the silhouette coefficients are lower for other subjects in this data set. Hence, the usefulness of a particular COP measure for differentiating between different stability conditions may be sufficient for some subjects, but, not necessarily for all subjects. Figure 6 shows similar inconsistent behavior for the standard deviation of $\mathrm{COP}_{x}\left(\sigma_{x}\right)$, standard deviation of $\mathrm{COP}_{y}$ $\left(\sigma_{y}\right)$, and standard deviation of radial displacement $S D_{-} R D$.

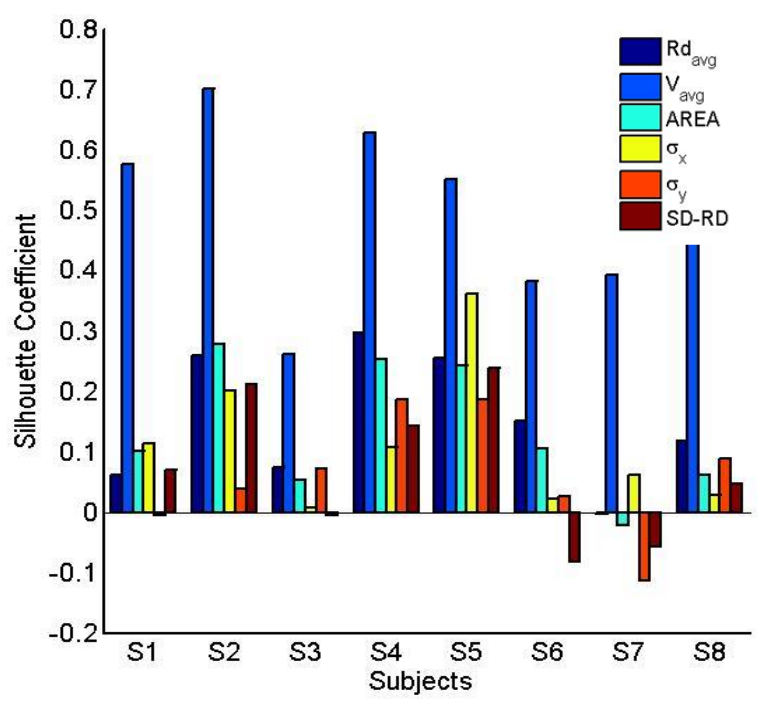

Fig. 6. Silhouette coefficient values for the COP measures for subjects \#1 to \#8.

\section{Conclusion}

The outcome of cluster analysis using the silhouette coefficient suggests that $V_{\text {avg }}$ is the best measure compared to other investigated conventional measures. The higher values of silhouette coefficients for $V_{\text {avg }}$ indicate that $V_{\text {avg }}$ for a given stability condition, are more repeatable (less variable) between trials from a data collection session, and more discernible between the different stability conditions. At the same time, this parameter can differentiate between different levels of stability conditions. Moreover, this study showed 
that cluster analysis provides a useful quantitative approach to compare the performance of different COP measures in different stability conditions.

These findings can assist in guiding future research to develop more strategies for early detection of instability in order to reduce the risk of falls in seniors and also individuals with balance disorders due to pathologies.

In this study, the performance of the six conventional measures are presented. There are other COP measures. More recent COP measures include approximate entropy (Cavanaugh et al., 2005), sample entropy (Donker et al., 2007), and the Lyapunov exponent (Donker et al., 2007). The approximate (Cavanaugh et al., 2005) and sample entropy (Donker et al., 2007) are used to measure the regularities of the time series and largest Lyapunov exponent (Donker et al., 2007) is a measure of local stability of a dynamical system. It is not evident which of these measures may have better clustering performance. Additional research is required to explore other measures that are reliable and useful for diagnostic purposes. The work presented here provides a strategy to quantitatively compare the performance of different COP measures and could be expanded to include other measures.

\section{Appendix}

\subsection{Measures of COP}

Define $x_{i}$ and $y_{i}$ as the zero-mean values of $C O P_{x}$ and $C O P_{y}$ for sample $i$ (the means of $C O P_{x}$ and $C O P_{y}$ subtracted accordingly). Define $N$ as the total number of samples.

Average Radial Displacement: The average radial displacement of the COP, $R D_{\text {avg }}$, is computed as

$R D_{a v g}=\frac{\sum_{i=1}^{N} r_{i}}{N}$

where $r_{i}=\sqrt{x_{i}^{2}+y_{i}^{2}}$; radial displacement for $i$ th sample.

Average Velocity: The average velocity of the COP, $V_{a v g}$, is computed as

$V_{a v g}=\frac{L}{N * \Delta t}$

where $L=\sum_{i=2}^{N} \sqrt{\left(x_{i}-x_{i-1}\right)^{2}+\left(y_{i}-y_{i-1}\right)^{2}}$ and $\Delta t=\frac{1}{f_{s}}$ with $f_{s}$ the sampling frequency.

Area of 95\% Ellipse of COP: The area of the 95\% ellipse of COP, $A R E A$, is computed as (Schmit et al., 2006).

$A R E A=(2 \pi \mathrm{F}) \sqrt{\sigma_{x}^{2} \sigma_{y}^{2}-\sigma_{x y}^{2}}$ where $F=3.00$ from the table of $F$ statistics at a confidence level of $1-\alpha$ with $\alpha=0.05$ when the sample size $>120$. Also, the correlation coefficient between $x_{i}$ and $y_{i}$ is given as

$\sigma_{x y}=\frac{1}{N} \sum_{i=1}^{N}\left(x_{i} * y_{i}\right) /\left(\sigma_{x} * \sigma_{y}\right)$.

Standard Deviation of $\operatorname{COP}_{x}(M L)$ : The standard deviation of $C O P_{x}, \sigma_{x}$, is calculated as

$\sigma_{x}=\sqrt{\frac{1}{N} \sum_{i=1}^{N}\left(x_{i}\right)^{2}}$

Standard Deviation of $\mathrm{COP}_{y}$ (AP): The standard deviation of $C O P_{y}, \sigma_{y}$, is calculated as

$\sigma_{y}=\sqrt{\frac{1}{N} \sum_{i=1}^{N}\left(y_{i}\right)^{2}}$

Standard Deviation of Radial Displacement: The standard deviation of radial displacement, $S D_{-} R D$, is calculated as

$S D_{-} R D=\sqrt{\frac{1}{N-1} \sum_{i=1}^{N}\left(r_{i}-R D_{a v g}\right)^{2}}$.

\section{References}

American Geriatrics Society, British Geriatrics Society, and American Academy of Orthopaedic Surgeons Panel on Falls Prevention. (2001). Guideline for the Prevention of Falls in Older Persons. Journal of the American Geriatrics Society, 49(5), 664-672.

American Geriatrics Society. (2001). Guideline for the Prevention of Falls in Older Persons. Journal of the American Geriatrics Society, 49(5), 664-672.

Askvik, B. (2010). Effects of exercise in elderly women with osteopenia or osteoporosis. Master Thesis in Sport Sciences, Department of Sports Medicine, Norwegian School of Sports Sciences.

Baig, S., Chan, A. D. C., Dansereau, R. M., Remaud, A., Bilodeau, M. (2012). Performance of COP measures. Proc. of International Conference on Electrical and Computer Systems, 194.1-194.8.

Baratto, L., Morasso, P. G., Re, C., Spada, G. (2002). A new look at posturographic analysis in the clinical context: swaydensity versus other parameterization techniques. Motor control, 6(3), 246-70.

Berg, K. O., Maki, B. E., Williams, J. I., Holliday, P. J., WoodDauphinee, S. L. (1992). Clinical and laboratory measures of postural balance in an elderly population. Archives of physical medicine and rehabilitation, 73(11), 1073-80.

Bickerstaffe, A., Beelen, A., Nollet, F. (2010). Circumstances and consequences of falls in polio survivors. Journal of rehabilitation medicine, 42(10), 908-15. 
Browne, J., O'Hare, N. (2000a). Development of a novel method for assessing balance: the quantitative posturography system. Physiological measurement, 21(4), 525-34.

Browne, J., O’Hare, N. (2000b). A quality control procedure for force platforms. Physiological measurement, 21, 515524.

Cavalheiro, G. L., Almeida, M. F. S., Pereira, A. A., Andrade, A. O. (2009). Study of age-related changes in postural control during quiet standing through linear discriminant analysis. BioMedical Engineering onLine, 8, 35.

Cavanaugh, J. T., Guskiewicz, K. M., Giuliani, C., Marshall, S., Mercer, V., Stergiou, N. (2005). Detecting altered postural control after cerebral concussion in athletes with normal postural stability. British journal of sports medicine, 39(11), 805-811.

Chaudhry, H., Bukiet, B., Ji, Z., Findley, T. (2011). Measurement of balance in computer posturography: Comparison of methods--A brief review. Journal of bodywork and movement therapies, 15(1), 82-91. Elsevier Ltd.

Clair, K. L., Riach, C. (1996). Postural stability measures: what to measure and for how long. Clinical biomechanics, $I I(3), 176-178$.

Curcio, C.-L., Gomez, F., Reyes-Ortiz, C. A. (2009). Activity restriction related to fear of falling among older people in the Colombian Andes mountains: are functional or psychosocial risk factors more important? Journal of Aging and Health, 21(3), 460-79.

Dietz, V. (1992). Human neuronal control of automatic functional movements: interaction between central programs and afferent input. Physiological reviews, 72(1), 33-69.

Donker, S. F., Roerdink, M., Greven, A. J., Beek, P. J. (2007). Regularity of center-of-pressure trajectories depends on the amount of attention invested in postural control. Experimental brain research. Experimentelle Hirnforschung. Expérimentation cérébrale, 181(1), 1-11.

Doyle, R. J., Hsiao-Wecksler, E. T., Ragan, B. G., Rosengren, K. S. (2007). Generalizability of center of pressure measures of quiet standing. Gait \& posture, 25(2), 166-171.

Duncan, P. W., Weiner, D. K., Chandler, J., Studenski, S. (1990). Functional reach: a new clinical measure of balance. Journal of gerontology, 45(6), M192-7.

Gage, W. H., Winter, D. A., Frank, J. S., Adkin, A. L. (2004). Kinematic and kinetic validity of the inverted pendulum model in quiet standing. Gait \& posture, 19(2), 124-32.

Gosselin, G., Rassoulian, H., Brown, I. (2004). Effects of neck extensor muscles fatigue on balance. Clinical biomechanics (Bristol, Avon), 19(5), 473-9.

Horak, F. B. (1987). Clinical measurement of postural control in adults. Physical therapy, 67(12), 1881-5.

Jbabdi, M., Boissy, P., Hamel, M. (2008). Assessing control of postural stability in community-living older adults using performance-based limits of stability. $B M C$ geriatrics, 8, 8.

Jensen, J., Nyberg, L., Gustafson, Y., Lundin-Olsson, L. (2003). Fall and injury prevention in residential care--effects in residents with higher and lower levels of cognition. Journal of the American Geriatrics Society, 51(5), 62735.

Lord, S. R., Menz, H. B., Tiedemann, A. (2003). A physiological profile approach to falls risk assessment and prevention. Physical therapy, 83(3), 237-52.

Massion, J. (1994). Postural control system. Current opinion in neurobiology, 4(6), 877-87.

Mcclenaghan, B., Williams, H. G., Dickerson, J., Dowda, M., Thombs, L., Eleazer, P. (1996). Spectral characteristics of aging postural control. Gait \& posture, 4, 112-121.

Nichols, D. S. (1997). Balance retraining after stroke using force platform biofeedback. Physical therapy, 77(5), 553-8.

Pellecchia, G. L., Shockley, K. (2005). Application of Recurrence Quantification Analysis: Influence of Cognitive Activity on Postural Fluctuations. In M. A. Riley \& G. C. Van Orden (Eds.), Tutorials in contemporary nonlinear methods for the behavioral sciences (pp. 95-141).

Prieto, T. E., Myklebust, J. B., Hoffmann, R. G., Lovett, E. G., Myklebust, B. M. (1996). Measures of postural steadiness: differences between healthy young and elderly adults. IEEE transactions on bio-medical engineering, 43(9), 956-66.

Raymakers, J. a, Samson, M. M., Verhaar, H. J. J. (2005). The assessment of body sway and the choice of the stability parameter(s). Gait \& posture, 21(1), 48-58.

Rousseeuw, P. J. (1987). Silhouettes: a graphical aid to the interpretation and validation of cluster analysis.pdf. Journal of Computational and Applied Mathematics, 20, 53-65.

Ruhe, A., Fejer, R., Walker, B. (2011). Center of pressure excursion as a measure of balance performance in patients with non-specific low back pain compared to healthy controls: a systematic review of the literature. European spine journal: official publication of the European Spine Society, the European Spinal Deformity Society, and the European Section of the Cervical Spine Research Society, 20(3), 358-68.

Schmit, J. M., Riley, M. a, Dalvi, A., Sahay, A., Shear, P. K., Shockley, K. D., Pun, R. Y. K. (2006). Deterministic center of pressure patterns characterize postural instability in Parkinson's disease. Experimental brain research, 168(3), 357-367.

Shimada, H., Suzukawa, M., Ishizaki, T., Kobayashi, K., Kim, H., Suzuki, T. (2011). Relationship between subjective fall risk assessment and falls and fall-related fractures in frail elderly people. BMC geriatrics, 11(1), 40. BioMed Central Ltd. 
Tinetti, M. E., Williams, T. F., Mayewski, R. (1986). Fall risk index for elderly patients based on number of chronic disabilities. The American journal of medicine, 80(3), 429-34.

Winter, D. A. (1995). Human balance and posture control during standing and walking. Gait \& posture, 3, 193214. 